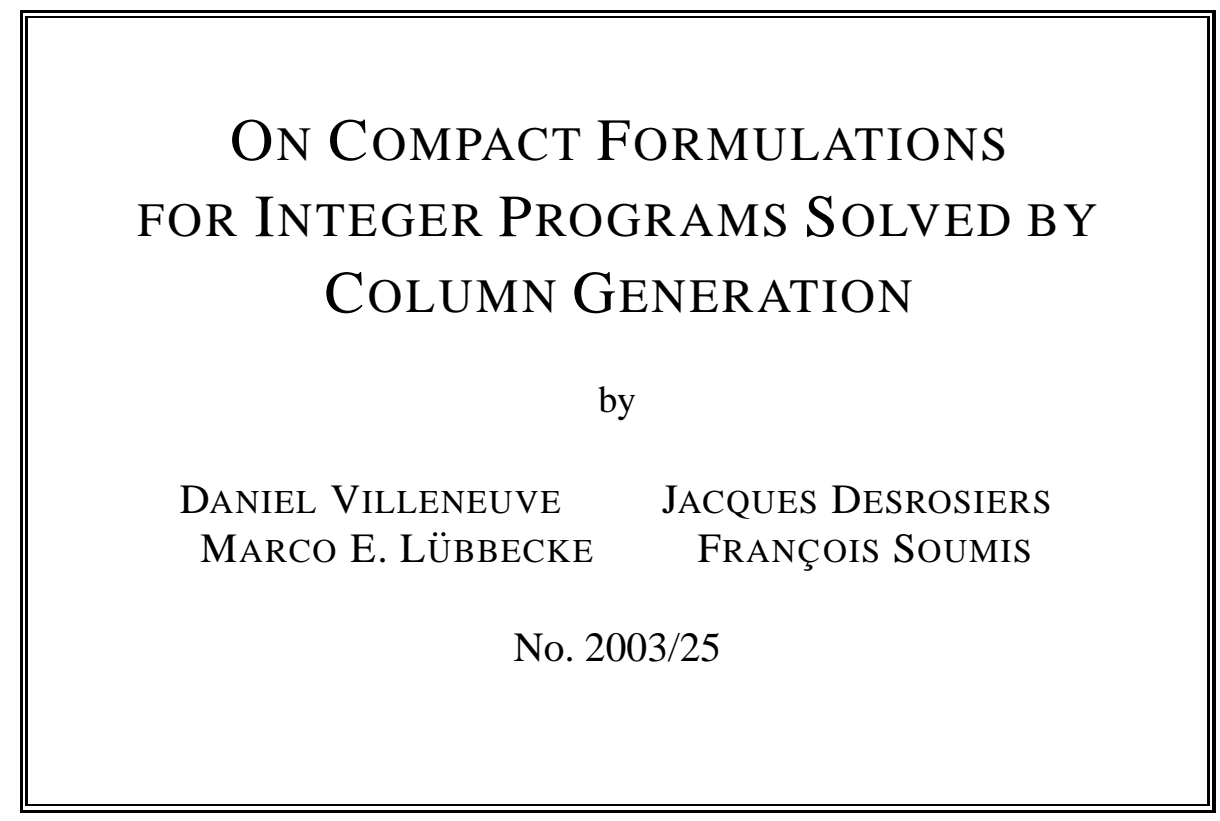




\title{
On Compact Formulations for Integer Programs Solved by Column Generation
}

\author{
Daniel Villeneuve \\ AD OPT Technologies Inc. \\ 3535 Queen Mary Road, Suite 650 \\ Montréal, H3V 1H8 Canada \\ Danielvead-opt.com
}

\author{
Jacques Desrosiers \\ HEC Montréal and GERAD \\ 3000, chemin de la Côte-Ste-Catherine \\ Montréal, H3T 2A7 Canada \\ Jacques.Desrosiers@hec.ca
}

\author{
Marco E. Lübbecke \\ Technische Universität Berlin \\ Institut für Mathematik, Sekr. MA 6-1 \\ Straße des 17. Juni 136, D-10623 Berlin, Germany \\ M. Luebbecke@math.tu-berlin.de \\ François Soumis \\ École Polytechnique de Montréal and GERAD \\ C.P. 6079, Succ. Centre-Ville \\ Montréal, H3C 3A7 Canada \\ Francois.Soumis@gerad.ca
}

December 9, 2003

\begin{abstract}
Column generation has become a powerful tool in solving large scale integer programs. It is well known that most of the often reported compatibility issues between pricing subproblem and branching rule disappear when branching decisions are based on imposing constraints on the subproblem's variables. This can be generalized to branching on variables of a so-called compact formulation. We constructively show that such a formulation always exists under mild assumptions. It has a block diagonal structure with identical subproblems, each of which contributes only one column in an integer solution. This construction has an interpretation as reversing a Dantzig-Wolfe decomposition. Our proposal opens the way for the development of branching rules adapted to the subproblem's structure and to the linking constraints.
\end{abstract}

Key words: Integer programming; column generation; branch-and-bound.

La méthode de génération de colonnes est devenue un outil important pour la résolution de très grands problèmes en nombres entiers. La plupart des difficultés de compatibilité rapportées dans la littérature entre les décisions de branchement et la résolution du sous-problème disparaissent lorsque les décisions sont basées les variables du domaine du sous-problème. Cette stratégie de branchement peut être généralisée au niveau des variables d'une formulation dite compacte. Sous de faibles hypothèses, nous montrons par construction qu'une telle formulation existe. Elle possède une structure bloc-diagonale où les sous-problèmes sont identiques, chacun ne contribuant que pour une seule colonne dans une solution entière. Cette construction peut s'interpréter comme le processus inverse de la décomposition de Dantzig-Wolfe. Cette approche ouvre de nouvelles perspectives sur le développement de règles de branchement adaptés à la structure du sous-problème et à celle des contraintes liantes.

Mots clés : Programmation en nombres entiers; génération de colonnes; arbre de séparation. 


\section{Introduction}

Branch-and-bound is a practically very successful generic method for solving mixed integer programs. It has been tailored to many particular applications, most notably by customized branching rules which exploit the respective problem structure. When the number of variables is huge, the bound at each node is obtained by column generation, that is, by iteratively adding variables via the questioning of an oracle (or subproblem or column generator, synonymously). The overall process, known as branchand-price or integer programming column generation, hinges on what is called the compatibility of the branching rules with the oracle. Our discussion puts the notion of compatibility into a new perspective.

Suppose a problem can be formulated in a compact way which explicitly reflects the oracle structure and a set of linking constraints. This type of formulation naturally leads to a solution by a decomposition process, such as the one proposed by Dantzig and Wolfe (1960). Therefore this problem can also be formulated in an extensive way, which results from the enumeration of a subset of solutions to the oracle. We make the point that the compact formulation does not only most naturally give rise to branching rules for the extensive formulation; its proper use also eliminates almost all difficulties with compatibility. Solving the compact formulation integrally is theoretically not different from solving any integer program, except for the way of computing the bounds by using the extensive formulation.

However, in particular in applications, it is not rare that only an extensive formulation and a pricing oracle are given. The contribution of this paper is to show, by construction, the existence of an associated compact formulation, and to demonstrate how it reduces compatibility issues.

Solving an extensive column generation formulation by way of a compact formulation can be seen as a straightforward, but useful complement of the use of the processes developed in the literature for decomposable integer programs, see e.g., Dantzig-Wolfe decomposition and Lagrangian relaxation. We outline how to algorithmically use the recovered compact formulation in integer programming column generation. The alternative we propose opens the way for the development of branching rules adapted to the oracle structure and to the linking constraints, in particular in a branch-and-cut context.

\section{Column Generation for Integer Programs}

Consider the following program which we call the compact formulation $C$ :

$$
v(C):=\min \left\{\mathbf{c}^{T} \mathbf{x} \mid A \mathbf{x}=\mathbf{b}, \mathbf{x} \in \mathcal{X}\right\} .
$$

This is an integer program for $\mathcal{X}=\mathcal{P} \cap \mathbb{Z}_{+}^{n}$ where $\mathcal{P} \subseteq \mathbb{R}^{n}$ is a polyhedron. We remark that $\mathcal{X}$ could have a much more complicated non-linear definition. Without loss of generality, we assume that $v(C)$ be finite. Replacing $\mathcal{X}$ by $\operatorname{conv}(\mathcal{X})$ does not change $v(C)$. It is well known (see Schrijver, 1986) that we can represent each $\mathbf{x} \in \operatorname{conv}(\mathcal{X})$ as a convex combination of extreme points $\left\{\mathbf{p}_{q}\right\}_{q \in \mathcal{Q}}$ plus a non-negative combination of extreme rays $\left\{\mathbf{p}_{r}\right\}_{r \in \mathcal{R}}$ of $\operatorname{conv}(\mathcal{X})$, where the index sets $\mathcal{Q}$ and $\mathcal{R}$ are finite, i.e.,

$$
\mathbf{x}=\sum_{q \in \mathcal{Q}} \mathbf{p}_{q} y_{q}+\sum_{r \in \mathcal{R}} \mathbf{p}_{r} y_{r}, \quad \sum_{q \in \mathcal{Q}} y_{q}=1, \quad \mathbf{y} \in \mathbb{R}_{+}^{|\mathcal{Q}|+|\mathcal{R}|}
$$

Substituting for $\mathbf{x}$ in (1) and applying the linear transformations $c_{j}=\mathbf{c}^{T} \mathbf{p}_{j}$ and $\mathbf{a}_{j}=A \mathbf{p}_{j}, j \in \mathcal{Q} \cup \mathcal{R}$, 
we obtain an equivalent extensive formulation $E_{C}$ of $C$ :

$$
\begin{aligned}
v\left(E_{C}\right):=\min \sum_{q \in \mathcal{Q}} c_{q} y_{q}+\sum_{r \in \mathcal{R}} c_{r} y_{r} & \\
\text { subject to } \sum_{q \in \mathcal{Q}} \mathbf{a}_{q} y_{q}+\sum_{r \in \mathcal{R}} \mathbf{a}_{r} y_{r} & =\mathbf{b} \\
\sum_{q \in \mathcal{Q}} y_{q} & =1 \\
\mathbf{y} & \geq \mathbf{0} \\
\mathbf{x} & =\sum_{q \in \mathcal{Q}} \mathbf{p}_{q} y_{q}+\sum_{r \in \mathcal{R}} \mathbf{p}_{r} y_{r} \\
\mathbf{x} & \in \mathbb{Z}_{+}^{n} .
\end{aligned}
$$

Typically, problem $E_{C}$ has a large number $|\mathcal{Q}|+|\mathcal{R}|+n$ of variables, but possibly substantially fewer rows than problem $C$. Equation $\sum_{q \in \mathcal{Q}} y_{q}=1$ is referred to as the convexity constraint over the extreme points of $\operatorname{conv}(\mathcal{X})$. This substitution easily generalizes to block diagonal matrices $A$, see Dantzig and Wolfe (1960). When we relax the integrality of $\mathbf{x}$, (2) becomes separable in $\mathbf{x}$ and $\mathbf{y}$, and we may also relax their linking constraints, obtaining a linear program in the variables $\mathbf{y}$ only. In general, requiring integrality of variables $\mathbf{y}$ does not lead to an integer program equivalent to $C$.

Alternatively, since the original variables $\mathbf{x}$ have to be integer (see also Holm and Tind, 1988), it is only natural to use them as the source of information in guiding branching and cutting decisions. Constraints representing these decisions on $\mathrm{x}$ are incorporated in $C$, either in the oracle structure or at the level of the linking constraints, and the decomposition process is then repeated. Problem $E_{C}$ is then used only to compute a lower bound and to identify a solution in terms of the relaxation of the compact formulation.

Consider now the following integer program $E$ formulated in an extensive way

$$
\begin{aligned}
v(E):=\min & \sum_{j \in J} c_{j} y_{j} \\
\text { subject to } & \sum_{j \in J} a_{i j} y_{j}=b_{i} \quad i \in I:=\{1, \ldots, m\} \\
y_{j} & \in \mathbb{Z}_{+} \quad j \in J
\end{aligned}
$$

the linear relaxation $E^{\prime}$ of which is supposedly solved by column generation using a given pricing oracle in any form, regardless of whether mixed integer program, combinatorial algorithm, or other. Observe that in general $E$ has no convexity constraint. We are not given an equivalent compact formulation on which we can analyze the solution of $E^{\prime}$, but we will show how to construct one. It has a block diagonal structure with identical subproblems. We also propose a general separation strategy, based on imposing constraints on the oracle's domain.

We can always assume that the finite set $J$ contains an index 0 for a dummy variable, i.e., $c_{0}=0$ and $\mathbf{a}_{0}=\mathbf{0}$. It is later used as a slack variable in the reformulation $C_{E}$ of $E$. We denote by $A=$ $\left\{\left(c_{j}, \mathbf{a}_{j}\right)\right\}_{j \in J}$ the set of all coefficient vectors. Note that this implies that $A$ contains no duplicate elements, i.e., there is an obvious bijection between $J$ and $A$. In column generation, the elements of $A$ are accessed using an oracle. We think of it as a surjective function $f: X \rightarrow A$ for some set $X$. The usefulness of the oracle relies on a smaller implicit description of $X$ compared to $A$, and on 
knowing an algorithm to compute a negative reduced cost column as in

$$
\min _{\mathbf{x} \in X} f_{0}(\mathbf{x})-\sum_{i \in I} u_{i} f_{i}(\mathbf{x})
$$

where $\mathbf{u} \in \mathbb{R}^{m}$ and $\boldsymbol{f}=\left(f_{0},\left(f_{i}\right)_{i \in I}\right)$.

\section{Solving $E$ by Branch-and-Bound}

\subsection{Bounding}

We obtain a lower bound on $v(E)$ from the linear programming relaxation $E^{\prime}$. The reduced cost $\bar{c}_{j}(\mathbf{u})=c_{j}-\sum_{i \in I} u_{i} a_{i j}$ of variable $y_{j}$ is defined as a function on the dual variables $\mathbf{u} \in \mathbb{R}^{m}$. Customarily, according to Dantzig's minimum reduced cost rule, the oracle returns a vector a $\in A$ corresponding to an index in $\arg \min _{j \in J} \bar{c}_{j}(\mathbf{u})$. Interestingly, not all elements $\mathbf{a} \in A$ can be generated this way. In the following example, if $c_{2}>1$, variable $z_{2}$ cannot be generated:

$$
\begin{aligned}
& \min z_{1}+c_{2} z_{2}+z_{3}
\end{aligned}
$$

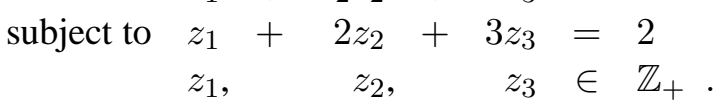

Given the dual variable $u \in \mathbb{R}$ associated with the equality constraint, $z_{2}$ is of minimum reduced cost if and only if $c_{2}-2 u \leq 1-u$ and $c_{2}-2 u \leq 1-3 u$, that is, if $c_{2} \leq 1-|u|$, in contradiction with $c_{2}>1$. It is a well known observation that the subset of generated columns may be integer infeasible. Here, we expose the stronger principal defect that even if feasibility could be ensured, sometimes we cannot obtain an optimal integer solution. This happens in (4) if $1<c_{2}<2$ : the unique optimal integer solution is $\left(z_{1}, z_{2}, z_{3}\right)=(0,1,0)$ of value $c_{2}$ while the solution restricted to the variables that can be generated is $\left(z_{1}, z_{3}\right)=(2,0)$ of cost $2>c_{2}$.

Similarly, Villeneuve (1999) illustrates that in Dantzig-Wolfe decomposition not all extreme points of the subproblem's polyhedron can be generated. We believe that analogous bad examples exist for other pricing rules as well. In conclusion, column generation may not only be necessary after branching, but also branching may be necessary to generate the right columns. Clearly, column generation has to be invoked not only at the root node but also at other nodes of the branch-and-bound tree. The next section shows various approaches reported in the literature which simultaneously take the pricing oracle's structure into account.

\subsection{Branching}

A branching rule partitions the solution space such that the current fractional solution is excluded, optimal integer solutions remain intact, and finiteness of the algorithm is ensured. The most immediate choice is to branch on variables of $E$. When the oracle supports excluding solutions which correspond to variables already down-branched on, this amounts to finding a $k^{\text {th }}$ best oracle solution instead of an optimal one (Sweeney and Murphy, 1979). This option is appealing because of its robustness against any restriction on $J$ whatsoever. On the downside is the increased complexity of the oracle which grows proportionally to the total number of variables excluded at the current node, which can be far greater than the depth of this node in the tree. When the oracle is for instance a shortest path problem, a pricing algorithm must not return paths which belong to a set of forbidden paths (Villeneuve and 
Desaulniers, 2002). It may not always be as easy as that and without taking care, the oracle structure can be destroyed by branching decisions (see e.g., Johnson, 1989; Savelsbergh, 1997; Vance, 1998).

An alternative to branching on single fractional variables of $E$ is to create two branches by imposing lower and upper bounds on a fractional sum of variables. When the oracle can be solved as an integer program, Vanderbeck (1994) proposes a strategy using a discretization of the oracle's domain. It is based on a rule originally developed by Ryan and Foster (1981) for set partitioning problems: Two rows must be covered either by one or by two distinct variables (see also Barnhart et al., 1998; Vanderbeck, 2000b). Vanderbeck and Wolsey (1996) generalize this to integer programs with general integer coefficients. Several other authors propose such a branching on constraints, either generic (branching on generalized upper bounds, or on special ordered sets, Nemhauser and Wolsey (1988)), or tailored to the respective oracle (e.g., Barnhart et al., 1997; Chen and Powell, 1999; Mehrotra et al., 2000; Mehrotra and Trick, 1996; Ryan and Falkner, 1987; van den Akker et al., 1999; Vanderbeck, 2000a). In general, the structure of the oracle has to be modified to take into account dual variables associated with the added branching constraints. Additional constraints may suffice, but more complex modifications may involve new constraints in $E^{\prime}$ as well as new binary variables and constraints in the oracle. The major drawback again is the increased size of the oracle integer program which might grow in some cases proportionally to the depth of the search tree.

The evident trade-off between a more elaborate branching rule and the resulting complication in the oracle leads to the notion of a compatible branching rule: The regeneration of variables which are forbidden in $E$ (by whatever rule) has to be avoided while not increasing too much (better: at all) the oracle complexity with the growing depth of the search tree. When the oracle allows for constraints in the form of bounds we may as well impose branching decisions to the oracle's domain. This is a natural support for branching on the original variables of a compact formulation, as is often implicitly done in rules which branch on constraints, see the references above. When branching rules and the oracle are not considered separately but in an integrated way, based on a common structure, the notion of compatibility becomes void. In fact, branching decisions reduce the oracle's complexity until its solution becomes trivial. The relaxation $E^{\prime}$ is then used for the purpose of bounding only and for identifying a solution in terms of the variables of the compact formulation.

This approach was originally proposed by Desrosiers et al. (1984) for a vehicle routing problem with time windows. Interestingly, there are two types of branching strategies in that paper: one applies directly to the flow variables of the oracle while the other imposes cuts on the total cost and the number of vehicles utilized. The link between both types is indeed the compact formulation where one finds the original decision variables (network flow and time variables), some of them being part of the linking constraints and the oracle structure. Multicommodity flow formulations for various applications of vehicle routing and crew scheduling proposed by Desaulniers et al. (1998) are similar to this scheme. Several other authors also use the same idea, see e.g., Desrochers et al. (1991); Desrosiers et al. (1995); Kohl et al. (1999); Sol (1994).

\section{A Reformulation $C_{E}$ of $E$}

We construct a compact formulation $C_{E}$ from which $E$ follows by application of a decomposition process. More precisely, Dantzig-Wolfe decomposition applied to $C_{E}$ yields an extensive formulation equivalent to $E$, however, with multiple column generators instead of the original single oracle we start

with. Each generator contributes only one column in a final integer solution. We restrict ourselves to the case where $X$ is closed and bounded, hence there exists a hyperbox $B$ such that $X \subset B \subset \mathbb{R}^{n}$. 
We assume that we know a $\kappa \in \mathbb{Z}_{+}$such that $\sum_{j \in J \backslash\{0\}} y_{j} \leq \kappa$ for a feasible solution y, i.e.,

$$
\sum_{j \in J} y_{j}=\kappa \quad \text { and } \quad y_{0}=\kappa-\sum_{j \in J \backslash\{0\}} y_{j} .
$$

We now formulate problem $C_{E}$ which explicitly reflects the functioning of the oracle:

$$
\begin{aligned}
& v\left(C_{E}\right):=\min \sum_{k \in K} f_{0}\left(\mathbf{x}^{k}\right) \\
& \text { subject to } \sum_{k \in K} f_{i}\left(\mathbf{x}^{k}\right)=b_{i} \quad i \in I \\
& \mathbf{x}^{k} \in X^{k} \quad k \in K:=\{1, \ldots, \kappa\} .
\end{aligned}
$$

We define for each $X^{k} \subseteq X$ a subset $J^{k} \subseteq J$ of indices associated with the set $f\left(X^{k}\right)$ of obtainable columns. We establish the connection between $E$ and $C_{E}$ via the following intermediate problem $\tilde{C}_{E}$, the variables $y_{j}^{k}$ of which split the variables $y_{j}$ of $E$ in $\kappa$ parts, i.e., $y_{j}=\sum_{k=g b 1}^{\kappa} y_{j}^{k}$ :

$$
\begin{aligned}
v\left(\tilde{C}_{E}\right):=\min \quad \sum_{k \in K} \sum_{j \in J^{k}} c_{j} y_{j}^{k} & \\
\text { subject to } \sum_{k \in K} \sum_{j \in J^{k}} a_{i j} y_{j}^{k} & =b_{i} \quad i \in I \\
\sum_{j \in J^{k}} y_{j}^{k} & =1 \quad k \in K \\
y_{j}^{k} & \in\{0,1\} \quad k \in K, \quad j \in J^{k} .
\end{aligned}
$$

Proposition 1 Problems $C_{E}$ and $\tilde{C}_{E}$ are equivalent.

Proof. Since $f$ is surjective, $|A|=|J|$ is finite, and $X^{k} \subseteq X, k \in K$, we may partition each $X^{k}$ into a finite number of equivalence classes, defined by $\mathbf{x}_{1}^{k} \equiv \mathbf{x}_{2}^{k}$ if and only if $\boldsymbol{f}\left(\mathbf{x}_{1}^{k}\right)=\boldsymbol{f}\left(\mathbf{x}_{2}^{k}\right)$. Taking a single representative per equivalence class we obtain a finite subset $\tilde{X}^{k} \subseteq X^{k}$ which we use instead of $X^{k}$ in (6). In order to reformulate $C_{E}$, let $\mathbf{x}_{j}^{k} \in \tilde{X}^{k}$ denote a representative of the equivalence class indexed by $j \in J^{k} \subseteq J$. By means of a binary linear combination of variables $y_{j}^{k}$ we express each $\mathbf{x}^{k} \in \tilde{X}^{k}, k \in K$ by exactly one of its $\left|J^{k}\right|$ representatives:

$$
\left\{\mathbf{x}^{k}=\sum_{j \in J^{k}} y_{j}^{k} \mathbf{x}_{j}^{k}, \quad \sum_{j \in J^{k}} y_{j}^{k}=1, \quad y_{j}^{k} \in\{0,1\}, j \in J^{k}\right\} \quad k \in K
$$

Using (8) we can write this change of variables in (6) as follows:

$$
\begin{array}{rlrl}
\min \sum_{k \in K} \sum_{j \in J^{k}} y_{j}^{k} f_{0}\left(\mathbf{x}_{j}^{k}\right) & & \\
\text { subject to } \sum_{k \in K} \sum_{j \in J^{k}} y_{j}^{k} f_{i}\left(\mathbf{x}_{j}^{k}\right) & =b_{i} & & i \in I \\
\sum_{j \in J^{k}} y_{j}^{k} & =1 & & k \in K \\
y_{j}^{k} & \in\{0,1\} & k \in K, \quad j \in J^{k} \\
\sum_{j \in J^{k}} y_{j}^{k} \mathbf{x}_{j}^{k} & =\mathbf{x}^{k} \in \tilde{X}^{k} & & k \in K .
\end{array}
$$


At optimality constraints $\sum_{j \in J^{k}} y_{j}^{k} \mathbf{x}_{j}^{k}=\mathbf{x}^{k} \in \tilde{X}^{k}, k \in K$ are not necessary since exactly one $\mathbf{x}_{j}^{k} \in \tilde{X}^{k}$ is picked on the left-hand side by the binary variables. With $f_{0}\left(\mathbf{x}_{j}^{k}\right):=c_{j}$ and $f_{i}\left(\mathbf{x}_{j}^{k}\right):=a_{i j}$, formulation (9) obtained by a change of variables in problem $C_{E}$ as given in (6) becomes identical to problem $\tilde{C}_{E}$ as given in (7).

Proposition 2 If $X^{k}=X$ for all $k \in K$, problems $\tilde{C}_{E}$ and $E$ are equivalent.

Proof. $X^{k}=X$ implies $J^{k}=J$. To complete the transformation, define variables $y_{j}=\sum_{k \in K} y_{j}^{k}$, $y_{j} \in \mathbb{Z}_{+}$. Summing up the $\kappa$ convexity constraints of $\tilde{C}_{E}$ yields

$$
\sum_{j \in J} \sum_{k \in K} y_{j}^{k}=\kappa \Longleftrightarrow \sum_{j \in J} y_{j}=\kappa,
$$

the last constraint being redundant by definition of $\kappa$ in (5). Therefore $\tilde{C}_{E}$ becomes

$$
\begin{array}{rlrl}
\min \sum_{j \in J} c_{j} y_{j} & & \\
\text { subject to } \sum_{j \in J} a_{i j} y_{j} & =b_{i} & & i \in I \\
y_{j} & \in \mathbb{Z}_{+} & & j \in J \\
y_{j} & =\sum_{k \in K} y_{j}^{k} & & j \in J \\
\sum_{j \in J} y_{j}^{k} & =1 & & \\
y_{j}^{k} & \in\{0,1\} & k \in K, \quad j \in J \\
\sum_{j \in J^{k}} y_{j}^{k} \mathbf{x}_{j}^{k} & =\mathbf{x}^{k} \in \tilde{X}^{k} & k \in K .
\end{array}
$$

It remains to be shown that $\tilde{C}_{E}$ given in (11) is equivalent to $E$ presented in (3). Clearly, any solution to (11) satisfies (3). On the other hand, we show that any solution to (3) in the non-negative integer variables $y_{j}$ can be split into $\kappa$ parts (allowing as many parts in variable $y_{0}$ as needed) which translate into variables $y_{j}^{k}$ of (11). By (10), at most $\kappa$ variables $y_{j}$ are positive. Without loss of generality, let $y_{0}, y_{1}, \ldots, y_{p}$ be these variables, with $y_{0}=\kappa-\sum_{j=1}^{p} y_{j}$. One possible assignment is:

$$
\begin{aligned}
y_{0}^{k} & = \begin{cases}1 & \text { if } 1 \leq k \leq y_{0} \\
0 & \text { otherwise }\end{cases} \\
y_{1}^{k} & = \begin{cases}1 & \text { if } y_{0}+1 \leq k \leq y_{0}+y_{1} \\
0 & \text { otherwise, }\end{cases} \\
\vdots & \\
y_{p}^{k} & = \begin{cases}1 & \text { if } \sum_{j=0}^{p-1} y_{j}+1 \leq k \leq \kappa \\
0 & \text { otherwise, }\end{cases} \\
y_{j}^{k} & =0 \text { if } j \notin\{1,2, \ldots, p\} \text { and } 1 \leq k \leq \kappa .
\end{aligned}
$$

Given this assignment, the $\mathrm{x}^{k}$-variables, $k \in K$ are trivially computed. Therefore problem $E$ is a relaxation of $\tilde{C}_{E}$, and the assertion follows. 
More generally, an integral assignment can be done using the constraints of a transportation problem. Note that for a solution to the linear relaxation of $E$ which is fractional in $y_{j}$, there exists a solution in $y_{j}^{k}$ that satisfies the linear relaxation of $\tilde{C}_{E}$. Indeed, assume that $y_{j}, j \in J$, is a solution to $E^{\prime}$ and assign to $y_{j}^{k}, k \in K, j \in J$, the values:

$$
y_{j}^{k}=\frac{y_{j}}{\kappa}, k \in K, j \in J
$$

By using (10), it is then easy to verify that this solution also satisfies $\tilde{C}_{E}^{\prime}$.

\section{Solving $E$ Using $C_{E}$}

By Proposition 1, a lower bound on $v\left(C_{E}\right)$ is provided by $v\left(\tilde{C}_{E}^{\prime}\right)$. Indeed, the linear relaxation $\tilde{C}_{E}^{\prime}$ is based on the convexification of each subset $X^{k}$ by using a convex combination of all feasible points in $X^{k}$ instead of the binary linear combination used in Proposition 1.

At a node in the branch-and-bound tree, when the solution of $\tilde{C}_{E}^{\prime}$ is integer, it replaces the incumbent solution if it improves on it. When $\tilde{C}_{E}^{\prime}$ is fractional, we make use of $\mathbf{x}^{k}=\sum_{j \in J^{k}} y_{j}^{k} \mathbf{x}_{j}^{k} \in \tilde{X}^{k}$, $k \in K$ to reconstruct a solution in terms of the variables of problem $C_{E}$. Since $X \subset B$, we define $B^{k}:=B, k \in K$ at the first node.

- If $\tilde{C}_{E}^{\prime}$ is fractional and $\mathbf{x}^{k} \notin X^{k}$ for some $k \in K$, we create two new nodes by dividing the hyperbox $B^{k}$ controlling the domain of $X^{k}$.

- If $\tilde{C}_{E}^{\prime}$ is fractional and $\mathbf{x}^{k} \in X^{k}$ for all $k \in K$, then the fractional combination of solutions of $X^{k}$ results in an integer solution for $C_{E}$. If $v\left(\tilde{C}_{E}^{\prime}\right)=v\left(C_{E}\right)$, i.e.,

$$
\sum_{k \in K} \sum_{j \in J^{k}} y_{j}^{k} f_{0}\left(\mathbf{x}_{j}^{k}\right)=\sum_{k \in K} f_{0}\left(\sum_{j \in J^{k}} y_{j}^{k} \mathbf{x}_{j}^{k}\right),
$$

the current solution is integer for $C_{E}$ (and $E$ ), and we explore the remaining nodes.

- If $\tilde{C}_{E}^{\prime}$ is fractional, $\mathbf{x}^{k} \in X^{k}$ for all $k \in K$, but $v\left(\tilde{C}_{E}^{\prime}\right)<v\left(C_{E}\right)$, the difference is reflected by some index $k \in K$. This may occur if the cost function $f_{0}$ is non-linear. In this case, we also create two new nodes by dividing the hyperbox $B^{k}$.

By successive reductions of $B^{k}, k \in K$ this branching process allows for the exploration of all solutions of problem $C_{E}$, an equivalent compact version of the extensive formulation $E$. We note that branching strategies are not limited to the bisection of $B^{k}, k \in K$. This strategy serves as a simple illustration on how to produce a valid search tree where the oracle does not become more complicated as branching decisions accumulate.

Problem $\tilde{C}_{E}^{\prime}$ may be huge, the number of variables compared to $E$ being increased by a factor of $\kappa$, plus the $\kappa$ convexity constraints. In the beginning, all subproblems are identical; hence a natural way to compute $v\left(\tilde{C}_{E}^{\prime}\right)$ is to aggregate the variables and the convexity constraints, and to use a single oracle. Subproblems may differ only after branching, and the corresponding convexity constraints are introduced as needed. 


\section{Applications}

In this section we present some applications, that is, the reconstruction of an adequate compact formulation on which branching and cutting strategies can easily be applied. Consider first the onedimensional cutting stock problem, a classical example in column generation. Given are paper rolls of width $W$, and $m$ demands $b_{i}, i \in I:=\{1, \ldots, m\}$ for orders of width $w_{i}$. The goal is to minimize the number of rolls to be cut into orders, such that the demand is satisfied. The standard extensive formulation by Gilmore and Gomory (1961), known for the strength of its linear relaxation bound, is

$$
\min \left\{\mathbf{1}^{T} \mathbf{y} \mid A \mathbf{y} \geq \mathbf{b}, \mathbf{y} \in \mathbb{Z}_{+}^{|J|}\right\},
$$

where $A$ encodes the set of $|J|$ feasible cutting patterns, i.e., $a_{i j} \in \mathbb{Z}_{+}$denotes how often order $i$ is obtained when cutting a roll according to $j \in J$. From the definition of the feasible patterns, condition $\sum_{i \in I} a_{i j} w_{i} \leq W$ must hold for every $j \in J$, and $y_{j}$ determines how often the cutting pattern $j \in J$ is used.

Given the dual multipliers $u_{i}, i \in I$, the linear relaxation of (12) is classically solved via column generation, where the pricing oracle is:

$$
\begin{aligned}
\min \quad\left(x_{0}-\sum_{i \in I} u_{i} x_{i}\right) & \\
\text { subject to } \quad \sum_{i \in I} w_{i} x_{i} & \leq W x_{0} \\
x_{0} & \in\{0,1\} \\
x_{i} & \in \mathbb{Z}_{+} \quad i \in I .
\end{aligned}
$$

In the above formulation, vector $\mathbf{x}=\left(x_{0},\left(x_{i}\right)_{i \in I}\right)$ separates in two parts: $x_{i}, i \in I$ is a non-negative integer variable that denotes the number of times order $i$ is cut in a roll, and $x_{0}$ is a binary variable assuming value 1 if a roll is used and 0 otherwise. Note that when $x_{0}$ is set to $1,(13)$ is equivalent to solving a knapsack problem while if $x_{0}=0$, then $x_{i}=0$ for all $i \in I$ and this null solution corresponds to an empty pattern, i.e., a roll that is not cut.

Our constructive procedure to recover a compact formulation equivalent to (12) leads to the definition of a specific subproblem for each roll. Let $K:=\{1, \ldots, \kappa\}$ be a set of rolls of width $W$ such that $\mathbf{1}^{T} \mathbf{y} \leq \kappa$ for some feasible solution $\mathbf{y}$. Let $\mathbf{x}^{k}=\left(x_{0}^{k},\left(x_{i}^{k}\right)_{i \in I}\right), k \in K$, be duplicates of the $\mathbf{x}$-vector, that is, $x_{0}^{k}$ is a binary variable assuming value 1 if roll $k$ is used and 0 otherwise, and $x_{i}^{k}, i \in I$ is a non-negative integer variable that denotes the number of times order $i$ is cut from roll $k$.

Defining $f_{0}\left(\mathbf{x}^{k}\right):=x_{0}^{k}$ and $f_{i}\left(\mathbf{x}^{k}\right):=x_{i}^{k}, i \in I$ the compact formulation (6) reads as follows:

$$
\begin{aligned}
\min \sum_{k \in K} x_{0}^{k} & \\
\text { subject to } \sum_{k \in K} x_{i}^{k} & \geq b_{i} \quad i \in I \\
\sum_{i \in I} w_{i} x_{i}^{k} & \leq W x_{0}^{k} \quad k \in K \\
x_{0}^{k} & \in\{0,1\} \quad k \in K \\
x_{i}^{k} & \in \mathbb{Z}_{+} \quad k \in K, i \in I .
\end{aligned}
$$

Formulation (14) of the cutting stock problem is due to Kantorovich (1960). It is known for the weakness of its linear relaxation. However, by applying an appropriate Dantzig-Wolfe decomposition, 
that is, in keeping the pricing oracle as an integer program given by (13), the lower bound provided by the linear relaxation of the resulting extensive formulation and that of (12) are the same. Branching decisions are then obviously taken on the $\mathrm{x}$-variables.

It should be pointed out that existence of a compact formulation does not mean uniqueness. There exist alternative compact formulations for the cutting stock problem that give rise to the same linear relaxation of the extensive formulation. Valério de Carvalho $(1999,2002)$ proposes a clever networkbased compact formulation in which the knapsack subproblem is solved as a particular minimum cost flow problem. Each subproblem path flow in that network gives a valid cutting pattern, and it corresponds to an extreme ray, except the null pattern which is the unique extreme point of the oracle's domain. We can turn the vast freedom of choosing a compact formulation into our advantage. For instance, we may wish to avoid symmetry (Barnhart et al., 1998) in the formulation. In fact, the process outlined in Section 4 is a good device in itself for reducing symmetry.

As a second type of application, consider column generation approaches used in the area of vehicle routing and crew scheduling. There the master problem is very often a set partitioning or a set covering problem while the oracle is given as a constrained shortest path problem. A path in the appropriate network represents a feasible itinerary for a vehicle, or for a crew. Therefore, it is only natural to define a specific subproblem for each vehicle or each crew member. This leads to (constrained) multicommodity flow problems as compact formulations, for which specialized branching and cutting decisions have been developed, see Desaulniers et al. (1998) for more details and the description of numerous branching and cutting rules. In the remainder we present two examples.

In Kohl et al. (1999), a subproblem is defined for each vehicle of the vehicle routing problem with time windows. The compact multicommodity flow formulation that is used allows cutting planes to be developed in terms of the network flow variables at the level of the linking constraints. These cuts generalize the subtour elimination constraints of the traveling salesman problem. Gamache et al. (1998) exploit in a special way the multicommodity formulation of their airline rostering problem. Here there is a subproblem for each pilot. The authors impose a very deep cut into selected subproblem domains. It does not only cut off the current infeasible fractional solution but at the same time it also removes from the oracle's domain a number of integer solutions.

\section{Conclusion}

Column generation and branch-and-bound are often reported to suffer from compatibility problems. Instead of considering these two components as separated we adopt a unifying perspective on solving extensive formulations by integer programming column generation. The principal advantage of solving an integer program by way of a compact formulation is to easily and directly exploit the structure of the oracle and that of the set of linking constraints. To a large extent the notion of compatibility becomes void in this framework. The small price we pay is the increased algorithmic administration of two concurrent formulations at a time.

Because of our rather general assumptions on the oracle there is quite some degree of freedom in obtaining a compact formulation, and imagination and experience are certainly helpful. Solving the compact formulation integrally is theoretically not different from solving any integer program, except for the way of computing the bounds by using the extensive formulation. This fundamental and indeed extremely simple approach has been in use now for almost twenty years (Desrosiers, Soumis, and Desrochers, 1984), and has been continually refined during this time. 


\section{Acknowledgments}

We would like to thank Hatem Ben Amor, Guy Desaulniers, and Stefan Irnich for the time they spent in discussing a preliminary version of this work, and pointing us to inaccuracies in the presentation. We also appreciate the constructive comments by two anonymous referees.

\section{References}

C. Barnhart, C.A. Hane, and P.H. Vance. Integer multicommodity flow problems. Lecture Notes in Economics and Mathematical Systems, 450:17-31, 1997.

C. Barnhart, E.L. Johnson, G.L. Nemhauser, M.W.P. Savelsbergh, and P.H. Vance. Branch-and-price: Column generation for solving huge integer programs. Oper. Res., 46(3):316-329, 1998.

Z.-L. Chen and W.B. Powell. Solving parallel machine scheduling problems by column generation. INFORMS J. Computing, 11:78-94, 1999.

G.B. Dantzig and P. Wolfe. Decomposition principle for linear programs. Oper. Res., 8:101-111, 1960.

G. Desaulniers, J. Desrosiers, I. Ioachim, M.M. Solomon, F. Soumis, and D. Villeneuve. A unified framework for deterministic time constrained vehicle routing and crew scheduling problems. In T.G. Crainic and G. Laporte, editors, Fleet Management and Logistics, pages 57-93. Kluwer, Norwell, MA, 1998.

M. Desrochers, J.K. Lenstra, M.W.P. Savelsbergh, and F. Soumis. Vehicle routing with time windows: Optimization and approximation. volume 16 of Studies in Management Science and Systems, pages 65-84. North-Holland, 1991.

J. Desrosiers, Y. Dumas, M.M. Solomon, and F. Soumis. Time constrained routing and scheduling. In M.O. Ball, T.L. Magnanti, C.L. Monma, and G.L. Nemhauser, editors, Network Routing, volume 8 of Handbooks in Operations Research and Management Science, pages 35-139. North-Holland, Amsterdam, 1995.

J. Desrosiers, F. Soumis, and M. Desrochers. Routing with time windows by column generation. Networks, 14:545-565, 1984.

M. Gamache, F. Soumis, D. Villeneuve, J. Desrosiers, and E. Gélinas. The preferential bidding system at Air Canada. Transportation Sci., 32(3):246-255, 1998.

P.C. Gilmore and R.E. Gomory. A linear programming approach to the cutting-stock problem. Oper. Res., 9:849-859, 1961.

S. Holm and J. Tind. A unified approach for price directive decomposition procedures in integer programming. Discrete Appl. Math., 20:205-219, 1988.

E.L. Johnson. Modelling and strong linear programs for mixed integer programming. In S.W. Wallace, editor, Algorithms and Model Formulations in Mathematical Programming, pages 1-43, Berlin, 1989. Springer. 
L.V. Kantorovich. Mathematical methods of organising and planning production. Management Sci., 6:366-422, 1960. Translation from the Russian original, dated 1939.

N. Kohl, J. Desrosiers, O.B.G. Madsen, M.M. Solomon, and F. Soumis. 2-Path cuts for the vehicle routing problem with time windows. Transportation Sci., 33(1):101-116, 1999.

A. Mehrotra, K.E. Murphy, and M.A. Trick. Optimal shift scheduling: A branch-and-price approach. Naval Res. Logist., 47(3):185-200, 2000.

A. Mehrotra and M.A. Trick. A column generation approach for graph coloring. INFORMS J. Comput., 8(4):344-354, 1996.

G.L. Nemhauser and L.A. Wolsey. Integer and Combinatorial Optimization. John Wiley \& Sons, Chichester, 1988.

D.M. Ryan and J.C. Falkner. A bus crew scheduling system using a set partitioning mode. Ann. Oper. Res., 4:39-56, 1987.

D.M. Ryan and B.A. Foster. An integer programming approach to scheduling. In A. Wren, editor, Computer Scheduling of Public Transport Urban Passenger Vehicle and Crew Scheduling, pages 269-280, Amsterdam, 1981. North-Holland.

M.W.P. Savelsbergh. A branch-and-price algorithm for the generalized assignment problem. Oper. Res., 45(6):831-841, 1997.

A. Schrijver. Theory of Linear and Integer Programming. John Wiley \& Sons, Chichester, 1986.

M. Sol. Column Generation Techniques for Pickup and Delivery Problems. PhD thesis, Eindhoven University of Technology, 1994.

D.J. Sweeney and R.A. Murphy. A method of decomposition for integer programs. Oper. Res., 27: 1128-1141, 1979.

J.M. Valério de Carvalho. Exact solution of bin-packing problems using column generation and branch-and-bound. Ann. Oper. Res., 86:629-659, 1999.

J.M. Valério de Carvalho. LP models for bin-packing and cutting stock problems. European J. Oper. Res., 141(2):253-273, 2002.

J.M. van den Akker, J.A. Hoogeveen, and S.L. van de Velde. Parallel machine scheduling by column generation. Oper. Res., 47(6):862-872, 1999.

P.H. Vance. Branch-and-price algorithms for the one-dimensional cutting stock problem. Comput. Optim. Appl., 9(3):211-228, 1998.

F. Vanderbeck. Decomposition and Column Generation for Integer Programs. PhD thesis, Université catholique de Louvain, 1994.

F. Vanderbeck. Exact algorithm for minimising the number of setups in the one-dimensional cutting stock problem. Oper. Res., 48(6):915-926, 2000a.

F. Vanderbeck. On Dantzig-Wolfe decomposition in integer programming and ways to perform branching in a branch-and-price algorithm. Oper. Res., 48(1):111-128, 2000 b. 
F. Vanderbeck and L.A. Wolsey. An exact algorithm for IP column generation. Oper. Res. Lett., 19: 151-159, 1996.

D. Villeneuve. Logiciel de Génération de Colonnes. PhD thesis, École Polytechnique de Montréal, 1999.

D. Villeneuve and G. Desaulniers. The shortest path problem with forbidden paths. Les Cahiers du GERAD G-2002-41, HEC Montréal, Canada, 2002. 
Reports from the group

\title{
"Combinatorial Optimization and Graph Algorithms"
}

\author{
of the Department of Mathematics, TU Berlin
}

2003/43 Michael R. Bussieck, Thomas Lindner, and Marco E. Lübbecke: A Fast Algorithm for Near Cost Optimal Line Plans

2003/42 Marco E. Lübbecke: Dual Variable Based Fathoming in Dynamic Programs for Column Generation

2003/37 Sándor P. Fekete, Marco E. Lübbecke, and Henk Meijer: Minimizing the Stabbing Number of Matchings, Trees, and Triangulations

2003/25 Daniel Villeneuve, Jacques Desrosiers, Marco E. Lübbecke, and François Soumis: On Compact Formulations for Integer Programs Solved by Column Generation

2003/24 Alex Hall, Katharina Langkau, and Martin Skutella: An FPTAS for Quickest Multicommodity Flows with Inflow-Dependent Transit Times

2003/23 Sven O. Krumke, Nicole Megow, and Tjark Vredeveld: How to Whack Moles

2003/22 Nicole Megow and Andreas S. Schulz: Scheduling to Minimize Average Completion Time Revisited: Deterministic On-Line Algorithms

2003/16 Christian Liebchen: Symmetry for Periodic Railway Timetables

2003/12 Christian Liebchen: Finding Short Integral Cycle Bases for Cyclic Timetabling

762/2002 Ekkehard Köhler and Katharina Langkau and Martin Skutella: Time-Expanded Graphs for FlowDependent Transit Times

761/2002 Christian Liebchen and Leon Peeters: On Cyclic Timetabling and Cycles in Graphs

752/2002 Ekkehard Köhler and Rolf H. Möhring and Martin Skutella: Traffic Networks and Flows Over Time

739/2002 Georg Baier and Ekkehard Köhler and Martin Skutella: On the $k$-splittable Flow Problem

736/2002 Christian Liebchen and Rolf H. Möhring: A Case Study in Periodic Timetabling

723/2001 Berit Johannes: Scheduling Parallel Jobs to Minimize Makespan

716/2001 Christian Liebchen: The Periodic Assignment Problem (PAP) May Be Solved Greedily

711/2001 Esther M. Arkin, Michael A. Bender, Sándor P. Fekete, Joseph S. B. Mitchell, and Martin Skutella: The Freeze-Tag Problem: How to Wake Up a Swarm of Robots

710/2001 Esther M. Arkin, Sándor P. Fekete, and Joseph S. B. Mitchell: Algorithms for Manufacturing Paperclips and Sheet Metal Structures

705/2000 Ekkehard Köhler: Recognizing Graphs without Asteroidal Triples

704/2000 Ekkehard Köhler: AT-free, coAT-free Graphs and AT-free Posets

702/2000 Frederik Stork: Branch-and-Bound Algorithms for Stochastic Resource-Constrained Project Scheduling

700/2000 Rolf H. Möhring: Scheduling under uncertainty: Bounding the makespan distribution 
698/2000 Sándor P. Fekete, Ekkehard Köhler, and Jürgen Teich: More-dimensional packing with order constraints

697/2000 Sándor P. Fekete, Ekkehard Köhler, and Jürgen Teich: Extending partial suborders and implication classes

696/2000 Sándor P. Fekete, Ekkehard Köhler, and Jürgen Teich: Optimal FPGA module placement with temporal precedence constraints

695/2000 Sándor P. Fekete, Henk Meijer, André Rohe, and Walter Tietze: Solving a "hard" problem to approximate an "easy" one: heuristics for maximum matchings and maximum Traveling Salesman Problems

694/2000 Esther M. Arkin, Sándor P. Fekete, Ferran Hurtado, Joseph S. B. Mitchell, Marc Noy, Vera Sacristán and Saurabh Sethia: On the reflexivity of point sets

693/2000 Frederik Stork and Marc Uetz: On the representation of resource constraints in project scheduling

691/2000 Martin Skutella and Marc Uetz: Scheduling precedence constrained jobs with stochastic processing times on parallel machines

689/2000 Rolf H. Möhring, Martin Skutella, and Frederik Stork: Scheduling with AND/OR precedence constraints

685/2000 Martin Skutella: Approximating the single source unsplittable min-cost flow problem

684/2000 Han Hoogeveen, Martin Skutella, and Gerhard J. Woeginger: Preemptive scheduling with rejection

683/2000 Martin Skutella: Convex quadratic and semidefinite programming relaxations in Scheduling

682/2000 Rolf H. Möhring and Marc Uetz: Scheduling scarce resources in chemical engineering

681/2000 Rolf H. Möhring: Scheduling under uncertainty: optimizing against a randomizing adversary

680/2000 Rolf H. Möhring, Andreas S. Schulz, Frederik Stork, and Marc Uetz: Solving project scheduling problems by minimum cut computations (Journal version for the previous Reports 620 and 661)

674/2000 Esther M. Arkin, Michael A. Bender, Erik D. Demaine, Sándor P. Fekete, Joseph S. B. Mitchell, and Saurabh Sethia: Optimal covering tours with turn costs

669/2000 Michael Naatz: A note on a question of C. D. Savage

667/2000 Sándor P. Fekete and Henk Meijer: On geometric maximum weight cliques

666/2000 Sándor P. Fekete, Joseph S. B. Mitchell, and Karin Weinbrecht: On the continuous Weber and kmedian problems

664/2000 Rolf H. Möhring, Andreas S. Schulz, Frederik Stork, and Marc Uetz: On project scheduling with irregular starting time costs

661/2000 Frederik Stork and Marc Uetz: Resource-constrained project scheduling: from a Lagrangian relaxation to competitive solutions

Reports may be requested from: $\quad$ Sekretariat MA 6-1

Fakultät II - Institut für Mathematik

TU Berlin

Straße des 17. Juni 136

D-10623 Berlin - Germany

e-mail: klink@math.TU-Berlin.DE 
Reports are also available in various formats from

http://www.math.tu-berlin.de/coga/publications/techreports/

and via anonymous ftp as

ftp://ftp.math.tu-berlin.de/pub/Preprints/combi/Report-number-year.ps 